\title{
Adaptation and collaborative CLTS pathways: Experiential learning from mistakes and iterative changes for success
}

\author{
Mr. Dektar Benbella (Corresponding Author) \\ Africa Centres for Disease Control and Prevention (Africa CDC), \\ Plot 13A, Suite 2 Reedbuck Road, Lusaka, Zambia \\ Email: dektarb@gmail.com \\ Mob. Tel. +260971 830820 \\ ORCID identifier: 0000-0002-5944-420X
}

\begin{abstract}
Background: This paper draws learnings and successes based on field implementation experience spanning four years (2016-2019) of implementing CLTS by Prince of Peace Orphans and Widows Vision, a community-based organization located in Kaberamaido district in eastern Uganda.

Methods: The study aimed to document, disseminate and inform from an evidence-based point of view how adaptation and collaborative engagements triggers learning from mistakes to inform iterative changes from improvements and success.

This is a descriptive paper that used project documents review based on field implementation experience. Existing project reports were synthesised, collated and curated for evidence. Data were drawn from project reports and records to inform narratives in writing. Implementation of the project was executed in homogenous rural communities occupied by people of the same dialect, cultural and social settings.

Results: We note that success in CLTS implementation can hardly be achieved by merely following prescriptions in handbooks and guidelines but rather by devising innovative community engagement and other participatory and community-driven techniques that foster adaptive management, promote ownership, and buy-in.

Having learned from our failures, we used data to inform decisions and transformatively deviated from traditional CLTS implementation and introduced high impact and innovative approaches such as the use of CLTS helpdesks and Situation room, the Pamoja approach, learning labs and iterative feedback loops, innovatively tackling slippage and carefully introducing the follow-up mandona approach. These enhanced experiential learning and ultimately resulted in sustained sanitation behaviour.

Conclusions: The CLTS approach as outlined in the handbook needs to be flexibly adapted to address contextual needs. Reflective and learning sessions reinforced with routine feedback loops from implementers and beneficiaries yields tremendous results, propagates experiential learning, and ultimately results in a transformative deviation from undesirable to desired sanitation behaviours. These innovative approaches once carefully blended have proved to be sustainable, are adaptable and can work in an even larger scale and in a variety of contexts.
\end{abstract}

Keywords

Adaptation, Failure, Feedback, Innovation, Learning, Sanitation, Scale-up, Success. 


\section{Contributions to the literature}

- This paper documents significant findings which explicate the use of innovative initiatives used in tandem to confront the crude facts around open defecation and its negative impacts on communities.

- The study addresses the paucity of information on implementation strategies carefully blended to improve community-based development outcomes in terms of sanitation improvement and attainment of open defecation free status.

- The study documents how community-based adaptive management practices steer communities to transformatively deviate to healthy behaviours.

- We also document how pluralistic engagement and grassroot collaboration fosters experiential learning by anchoring decision-making organs at various administrative levels of local governance.

\section{Background}

Traditionally, sanitation has not received the priority it deserves. It has not been widely recognized how good sanitation policies and practices can underpin socio-economic development and environmental protection (WSP, 2012).

Rural sanitation coverage in Uganda currently stands at $77.2 \%$, and district reports show that $22.9 \%$ of the rural population practice open defecation. Kaberamaido district household sanitation coverage stands at $93.8 \%$ while handwashing- the proxy for measurement of hygiene (presence and use of handwashing facilities) stands at 51.9\% (MWE, 2019). In 2015, sanitation coverage was 77\% in Kaberamaido district (MWE, 2015).

This paper is based on field implementation experience of the Kaberamaido sanitation and hygiene initiative (KASH) project implemented for four years (2016-2019) with a goal of allyear-round access to sanitation facilities and sustained behaviour change. Community-led total sanitation (CLTS) was the core approach used for sanitation promotion. The project was 
implemented by prince of peace orphans and widows vision (POPOW) in Kaberamaido district located in Eastern Uganda, targeting 40 villages; 10 in each of the four priority sub-counties. This study specifically set out to to document, disseminate and inform from an evidence-based point of view how adaptation and collaborative engagements triggers learning from mistakes to inform iterative changes from improvements and success

The project used a phased approach of implementation targeting five villages per sub-county and later scaling up to 10 villages per sub-county after drawing lessons learned from the first five.

The paper addresses traditional CLTS implementation pitfalls by the use of innovative learning mechanisms such as the Pamoja approach, CLTS champions, and community-based CLTS helpdesks, which offer opportunities for iterative reflection and learning ultimately resulting in local ownership, evidence-based adaptation and eventual scale-up of interventions in a sustainable fashion.

\section{Methods}

This was a descriptive paper which draws narratives from the community-led total sanitation (CLTS) interventions implemented by Prince of Peace Orphans and Widows Vision (POPOW), a community-based organization (CBO) located in Kaberamaido district (Figure 1) in eastern Uganda approximately 390 kilometres from Kampala city, the capital of Uganda. 


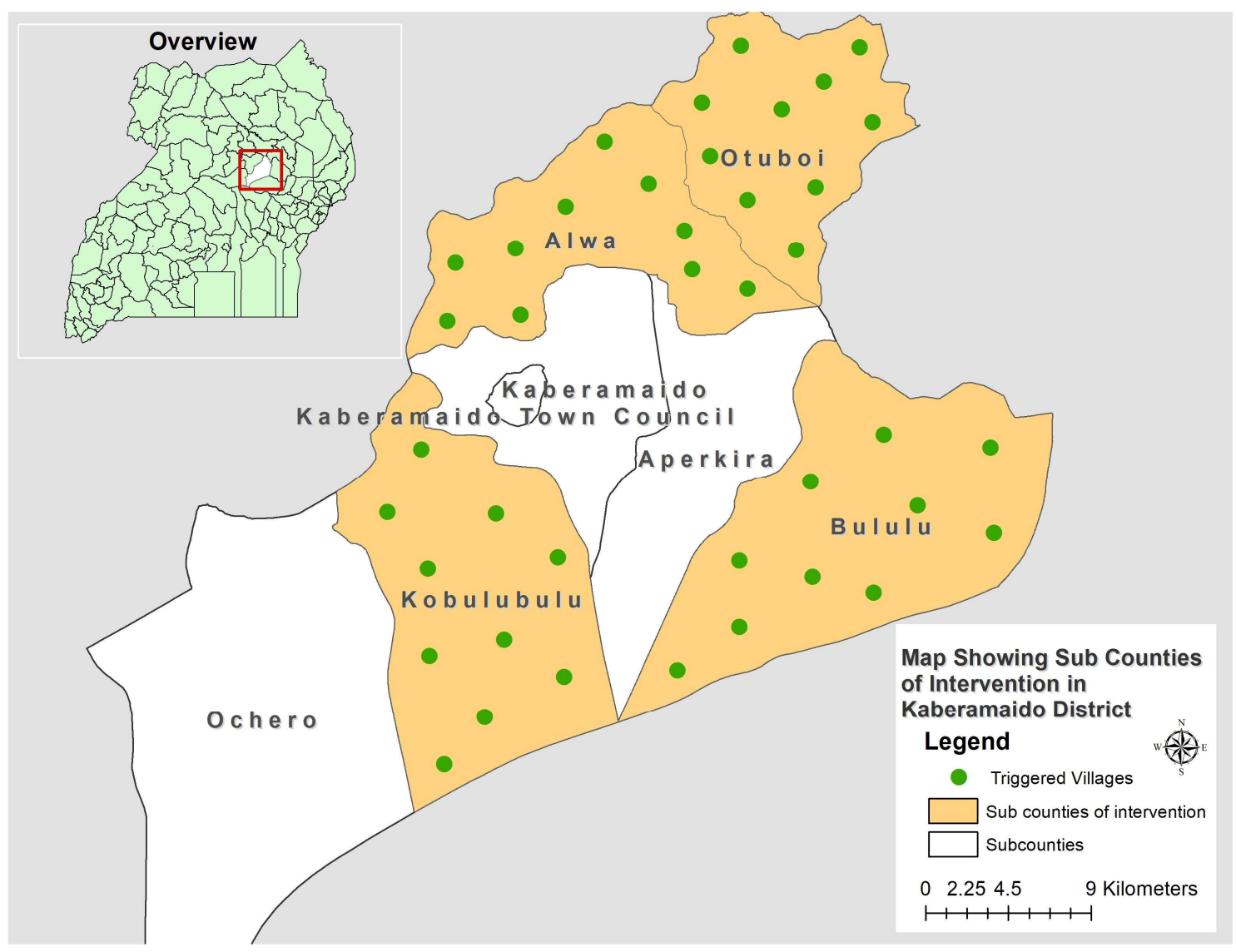

Figure 1: Sub-counties of intervention and triggered villages

Implementation of the project was executed in homogenous rural communities occupied by people of the same dialect, cultural and social settings.

This is a descriptive paper that used project documents review and implementation experience to draw its learnings and conclusions. Data were drawn from project reports and records to inform narratives in writing.

The next section presents objective and critical learning points drawn from this CLTS implementation experience.

\section{Results}

Records of CLTS implementation were obtained from project reports and other documents.

The data depicts mean sanitation coverages for the 10 triggered villages in each sub-county 
(Table 1). The baseline (2016) and final (2019) sanitation coverages after four years of the CLTS project are indicated.

\begin{tabular}{llccccc}
\hline$\#$ & Sub-county & Population & $\begin{array}{c}\text { Total \# } \\
\text { of } \\
\text { villages }\end{array}$ & $\begin{array}{c}\text { \# of villages } \\
\text { triggered }\end{array}$ & $\begin{array}{c}\text { 2016 } \\
\text { Sanitation } \\
\text { Coverage (\%) }\end{array}$ & $\begin{array}{c}\text { 2019 } \\
\text { Sanitation } \\
\text { Coverage (\%) }\end{array}$ \\
\hline 1. & Alwa & 23,332 & 49 & 10 & $68 \%$ & $94 \%$ \\
2. & Bululu & 21,390 & 41 & 10 & $62 \%$ & $96 \%$ \\
3. Kobulubulu & 19,658 & 38 & 10 & $71 \%$ & $91 \%$ \\
4. & Otuboi & 25,953 & 43 & 10 & $58 \%$ & $89 \%$ \\
\hline
\end{tabular}

Table 1: Baseline and final sanitation coverages in sub-counties of intervention (Source: Unpublished observations)

A trend analysis was conducted to ascertain the progress in household sanitation improvement across the target sub-counties from 2016 through 2019 (Figure 2)

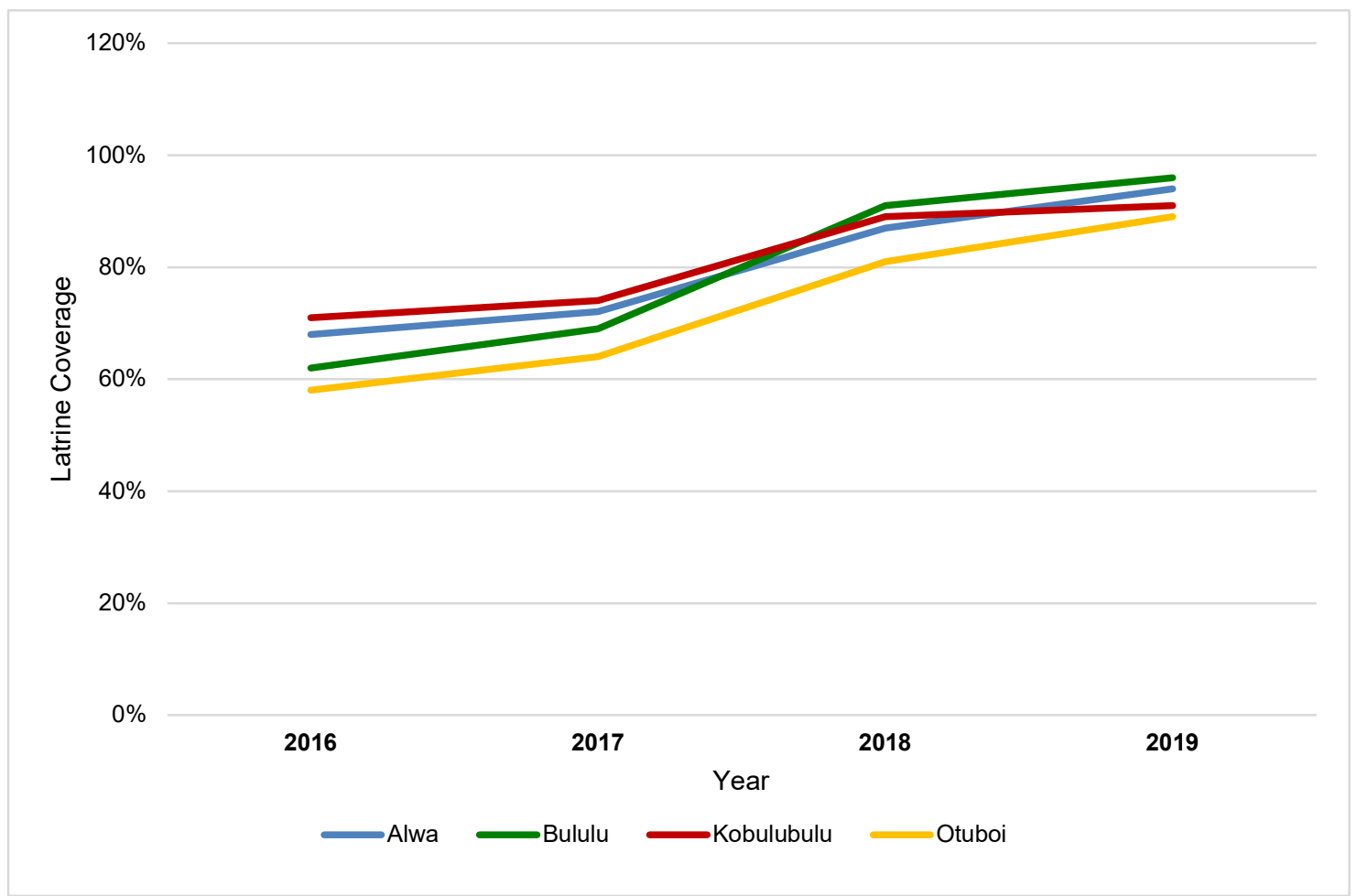

Figure 2: Household sanitation progress in sub-counties of intervention

From the above figure, we notice a steady and progressive trend in sanitation coverage over time. Whereas there was slow progress between 2016 and 2017, we see a sharp rise from 2017 
to 2018. The sharp increase is attributed to the implementation of innovative community engagement approaches, having learned from our failures in the first half of the project. The data shows the average sanitation coverages of the 10 villages triggered in each of the four subcounties. The selection criteria were such that; the villages targeted and selected met the CLTS favourable conditions, had the least sanitation coverages and did not benefit from any other water, sanitation, and hygiene (WASH) interventions from other organisations or government grants at the time of the needs assessment in 2016.

\section{Lessons from traditional CLTS triggering and follow-ups}

It was common practice for us to simply follow the guidelines as prescribed in the Handbook on Community-led Total Sanitation (Kar, 2008) without thinking outside the box. As such, we conducted CLTS in a 'business as usual' fashion with little or no results even when we had well trained and certified CLTS facilitators among our staff. As the manual illustrates, we simply followed through, triggering communities and returning to those communities for traditional follow-ups after three or four weeks and, in some cases, not returning for follow-up at all. Our follow-ups were largely conducted by the project team alone without involvement of the natural leaders who emerged during the triggering sessions nd neither were local leaders and other elders involved. With this kind of approach, we got no tangible results, experienced a slow improvement in sanitation coverage, and could hardly declare any village open defecation free (ODF) even after nearly a dozen rounds of follow-ups. Moreover, our follow-ups were not participatory and thus lacked the much-needed pluralistic engagement since we hardly involved stakeholders such as community leaders and local government field extension staff. In other words, our stakeholder and community engagement styles, too, were weak and not engaging. Following one year of implementation, after no tangible results and success, we held a reflection meeting to overhaul our approach to CLTS and introduce action-oriented and high impact approaches to our sanitation programming. This gave birth to establishing a CLTS 
Helpdesk and Situation Room at the office. The information helpdesk manned by a CLTS facilitator served as a one-stop-shop for CLTS information and advice to people in the triggered villages. At the same time, the Situation Room took stock of weekly progress in sanitation and hygiene status to identify villages progressing at a slow pace so that we could make efforts to reach them promptly. Later, we also introduced additional approaches, including a household clustering approach dubbed 'Pamoja' (Swahili word for together) which helped reinforce the follow-up mandona (FUM) approach. These approaches are described below. On their own, communities ably started to implement quick wins and actions which were within their locus of control and manageable interest.

\section{CLTS information Helpdesk and Situation Room}

The information helpdesk and situation room worked quite well in the first four months, with community members calling the helpline and regularly visiting to obtain information and reporting challenges at hand. However, we noticed a reduction in the number of calls and visitations to the helpdesk located at the office. Community meetings revealed that project beneficiaries had difficulties accessing the office due to poor roads, which are impassable during rainy seasons. Obtaining money to hire motorcycles or bicycles from their villages to the office to collect information and report sanitation progress data was a challenge with a lack of funds to purchase airtime for telephone calls to the helpdesk notwithstanding.

From these lessons and having noticed a slack in data provision from the communities, we decided to move the CLTS information helpdesks closer to the communities for easy access and consultation and ensure timely data gathering for the Situation Room, which remained stationed at the office. The helpdesks were later established in a central village to all those which had been triggered. This worked perfectly well as the Helpdesks at community levels were manned by trained individuals (CLTS champions) who were residents of the beneficiary villages. 
To strengthen their capacities and skillsets, refresher pieces of training were held for the community-based CLTS facilitators, and those who shone and performed better in sanitation improvement in their villages/ zones were elevated to 'CLTS champions' whose roles were to man the CLTS helpdesk and relay data to the Situation Room weekly among other responsibilities.

\section{The Pamoja approach}

In the third year of implementation, we noticed changes in community dynamics and context, and based on available information, we introduced a more innovative and collaborative approach to improve sanitation coverage. The Pamoja approach is a household clustering method that reinforces the FUM approach. Households worked together to help each other construct latrines beginning with the most vulnerable families, such as child-headed households, people living with disabilities, and the elderly. This approach was embraced and received high acceptance by the communities and was one of the most effective as we noticed a rapid increase in sanitation coverage across all triggered villages. The sharp rise in sanitation coverage depicted in figure 2 above is attributable to the shift in the way we managed the helpdesk by making them community-based, and the Pamoja approach. The Pamoja approach led to lateral diffusion (self-triggering) in two villages. Since the two were targeted by the project for intervention, having met the favourable conditions for CLTS, we skipped the triggering sessions and initiated the Pamoja approach. Besides, we also initiated other innovative techniques such as learning labs to ensure that learning and adaptation were intentional.

\section{Learning Labs (Iterative reflection sessions, and feedback loops)}

Having learned from our failures and successes in CLTS over the years, we were able to adapt interventions basing on information, data, contextual changes, and community dynamics. Through collaborative engagements with stakeholders and partners such as the local 
governments at district and sub-county levels, a mechanism of routine fortnightly meetings was established to reflect on our approaches and methods and tease out those which were working and why they were. We also used these reflection sessions to assess which approach was slow or not working and why. To ensure complete feedback loops, information was given to beneficiaries, but we also received feedback/ information in return. The Learning Labs were useful platforms for reflection with beneficiaries who often took the lead and suggested alternatives in our approaches. In essence, these community engagements and consultation meetings on CLTS, as well as the FUM approach, were vital in ensuring success and eventual ODF declaration in 37 out of the targeted 40 villages triggered. This translates to a 93\% ODF success rate. The FUM approach entails undertaking a series of sessions with triggered communities to enable them to initiate small, immediate, and doable actions within their control which will propel them to ODF status in the shortest possible time.

Some of the changes and adaptations we made courtesy of the Learning Lab reflections included; moving the CLTS helpdesks closer to communities thus rendering them communitybased and community-managed, a shift from 'project staff-only' follow-ups to participatory follow-ups involving stakeholders, and increasing the frequency of follow-ups from monthly to weekly among other changes.

\section{Tackling slippage (fall-back or retreat to open defecation)}

In general terms, slippage refers to the return to previous unhygienic behaviours or the inability of some or all community members to continue to meet all ODF criteria (Jerneck et al., 2016). Slippage was one of the challenges we had to deal with. It was primarily caused by technological and poverty vulnerability factors. Technological factors were mainly issues around latrine durability, materials, and design. The introduction of CLTS champions the majority of whom doubled as local sanitation artisans (masons) helped tackle the issue. To effectively tackle slippage, champions who bore masonry skills used their expertise to reinforce 
existing traditional pit latrine superstructures and roofs thereby rendering them more durable. In many cases, they also took up the construction of better and durable pit latrines for needy households at subsidised costs. There was a multiplier effect and skills transfer as champions with masonry skills trained others from neighbouring villages on the basics of bricklaying and durable latrine construction. Besides, the Pamoja approach was vital in addressing vulnerability factors around poverty, old age, child-headed households, people living with disabilities, and those affected by terminal illnesses.

\section{Use of post triggering after-action reviews}

After numerous failures in year one, we learned that we hardly made time to sit as a team and reflect on the triggering outcomes. As such, we barely had opportunities to address emerging issues as well as take stock of what went well and what didn't, what we needed to keep and what needed to be discontinued.

After scaling up to another 20 villages, after-action review (AAR) was a mandatory step conducted immediately after the triggering and follow up mandona sessions. This was helpful in the sense that we were able to learn from our mistakes and what could have gone wrong during the triggering events. We then used these AAR sessions to come up with immediate remedial strategies to address the pitfalls. As a result, we were able to adapt our CLTS interventional pathways and approaches based on data and available information. Whereas the Learning Lab sessions were community-driven with the participation of a wide range of stakeholders, AAR sessions were internal and participation was restricted to only project staff who participated in the triggering event.

\section{Discussion}

The results of this study are reflective of evidence-based failure-to-success documentation. Review of on-the-ground implementation of sanitation behaviour change advanced using the impactful Community-led Total Sanitation (CLTS) approach provided the much-needed 
context reinforced by implementation experience. Moreover, the involvement of a local community-based organisation strengthened the credibility of data and information used and fortified results discussed herein.

Implementation of development assistance programmes is often challenging in rural establishments, given the socio-cultural dynamics, poverty, and dependency syndrome.

The failures experienced in the initial years of implementation are attributable to our ineptitude in identifying rapidly-changing community dynamics and not taking time to reflect on the outcomes of the interventions and initiatives. We, therefore, had to make iterative adaptations and modifications as described in the findings to overhaul and salvage the project from failure. This is consistent with Greaves (2016), who reported that contexts, and other emerging programming themes, may appear to make CLTS more challenging to implement effectively. This calls for more learning about the adaptation of the CLTS process to different settings and diversification of the approach.

The CLTS approach is designed to enable rural open defecators to confront the negative repercussions of this behaviour by triggering collective behaviour change and facilitating collective action to adopt safe and hygienic sanitation behaviour.

Based on the results of this study, we noticed a surge in latrine coverage from a mean of $69.8 \%$ to $87.0 \%$ in 2017 and 2018 , respectively. These results peaked at a mean of $92.5 \%$ at the end of the intervention. With the blend of innovative initiatives such as CLTS learning labs, situation room, and CLTS helpdesks, 93\% (37/40) of the triggered villages attained ODF status. This provides evidence that the CLTS approach is instrumental in guiding villages to the desired sanitation status once used effectively. A study documented by USAID concurred with this finding, stating that; the current literature points to the ability of CLTS to generate significant short-term reductions in open defecation as well as increases in latrine coverage and use (USAID, 2018). 
The general increment in latrine coverage from baseline in 2016 to 2019 saw an average increase of $27.8 \%$ in latrine coverage over four years. This finding surpasses the average of $12 \%$ documented in literature and therefore is in line with a study that reported that the success of CLTS could be measured by latrine coverage, the percentage of households within a community that have access to their own latrines. A literature review of sanitation campaigns has reported an average increase in latrine coverage following CLTS of $12 \%$, though not statistically significant (Garn et al, 2016).

Upon completion of the project, the mean latrine coverage across all the sub counties was 92.5\%. This corroborates with and Robinson (2016), whose study presented up to $96 \%$ latrine coverage in a single case in Malawi.

In the intervention, the integration of other small and doable actions as well as intentional and meaningful community involvement were the game-changers. This is in sync with a study that was in agreement with initiating additional actions hence suggesting that this study corroborates the effectiveness of CLTS in increasing latrine coverage, and additional activities can be improved further (Harter, et al, 2020).

Although we had numerous successes to celebrate, there were some challenges to deal withslippage was one major factor we grappled with. This was primarily due to poverty vulnerability factors as wells as technological challenges. Whereas the use of CLTS champions was instrumental in addressing slippage, the challenge continues to lay bare the strength of the CLTS approach in sustaining sanitation behaviour change. This finding agrees with WHO (2018) which reported that its success rates vary widely across projects and countries and slippage rates as well as long-term effects have received too little scientific attention so far (WHO, 2018).

The CLTS Helpdesks were real-time, given that toll-free lines numbers were used to enable communities to reach out for information and advise and reports challenges without having to 
worry about airtime. This initiative helped a great deal in bridging the gap between the project and its beneficiaries. A similar approach reported by USAID through its LEARN mechanism was used in Zambia to ensure corrective actions were taken in a timely fashion.

The DHIS2-based Mobile-to-Web (M2W) monitoring system has been a critical element for Community Approaches to Total Sanitation (CATS) success from the start. The system has increased accountability, enabled feedback and course correction, and generated healthy competition between chiefs and districts. Accountability has increased because it is now evident which community champions (local volunteers) are consistently following up with their communities and which ones may be inactive and may need support or replacement (USAID LEARN, 2018).

To our knowledge, there are not as many studies that document a blend of innovative actions simultaneously incorporated into the traditional CLTS processes. Moreover, not as many studies describe how these work in tandem to break sanitation barriers and influence behaviour change. This study, therefore, lays a firm foundation upon which learnings can be drawn not only for replication but also scale up and innovation in rural sanitation programming anchoring the CLTS model in the approach.

Overall, our results indicate that for the CLTS model to be beneficial, incorporating innovative initiatives and reinforcing community engagement are absolute imperatives.

\section{Conclusion}

On a conclusive remark, it is worth mentioning that, the CLTS approach as outlined in the CLTS Handbook needs to be flexibly adapted to address contextual needs to ensure universal health coverage so that no one is left behind as far as sanitation service delivery is concerned. Thinking outside the box and introducing other innovative techniques plays a vital role in improving rural latrine coverage. Having reflective and learning sessions reinforced with routine feedback loops from implementers and beneficiaries yields tremendous results, propagates experiential learning, 
and ultimately results in a transformative deviation from undesirable to desired sanitation behaviours. Besides, using available information and data to review performance, existing approaches being used are critical in fostering adaptive management in sanitation promotion and programming, ultimately strengthening stakeholder collaborative pathways and sustainable sanitation behaviour change. Based on our experience and learnings, for good results, we recommend that implementers adopt the approaches documented in this paper but carefully adapt them based on contextual needs within their communities of intervention.

Having proven and tested these approaches over the past three years in one district, with stronger commitment and support from district local governments, these approaches are adaptable and can work on an even larger scale and in a variety of contexts within the country, more so since the helpdesks, Situation Room, and Pamoja are being sustained to date even after project closeout.

\section{List of abbreviations}
AAR: $\quad$ After Action Review
CBO: $\quad$ Community-based Organization
CLTS: $\quad$ Community-led Total Sanitation
FUM: $\quad$ Follow-up Mandona
ODF: $\quad$ Open Defecation Free
PI: $\quad$ Principal Investigator
POPOW: $\quad$ Prince of Peace Orphans and Widows Vision
WASH: Water Sanitation and Hygiene

\section{Declarations}

\section{Ethics approval and consent to participate}

This study was based on field implementation experience so did not require approval from an ethical committee or other scientific body. However, permission to conduct the stuy and access 
project records, data and statistics was obtained from the leadership of Prince of Peace Orphans and Widows Vision. In so doing, as the principal investigator (PI), I explained the purpose, objectives, risks and benefits of the study, and how data obtained from project records will be utilised to derive findings.

\section{Consent for publication}

Not Applicable

\section{Availability of data and materials}

The methodology of the study did not warrant collection of primary data but rather relied on secondary data obtained from project reports and records. The statistics presented in the table was based on this. On reasonable request, the dataset used and/or analyzed for purposes of accomplishing this study, can be availed by the PI of this publication.

\section{Competing interests}

The author declares that he has no competing interests.

\section{Funding}

This work was not funded by any entity. The decision to publish came from the author of the study.

\section{Authors' contributions}

Only one author was involved in the entire study. The sole author designed the study, analysed data, and interpretation of results, manuscript drafting and revision.

\section{Acknowledgements}

The author expresses sincere gratitude to POPOW staff and local government extension workers for their valuable contributions and support rendered in digging out project reports for data and information used to enrich this paper. Finally, the author would like to declare that the views expressed in this publication do not necessarily represent the views, decisions, or policies 
of the implementing agency (POPOW), institution with which he is affiliated, only the author is responsible for these views.

\section{Author details}

About the author is a Public Health practitioner with a profound interest in community-based rural sanitation promotion. He is also passionate about documentation, monitoring, measurement, evaluation and collaborative learning and adaptive management in water and sanitation programs and other development assistance programs.

Benbella Dektar, Africa Centres for Disease Control and Prevention (Africa CDC),

Plot 13A, Suite 2 Reedbuck Road, Lusaka, Zambia

Telephone: +260 971830 820. Email: dektarb@gmail.com

\section{References}

1. Jerneck M, Voorden C, Rudholm C. Sanitation and Hygiene Behaviour Change at Scale: Understanding Slippage, Geneva: Water Supply and Sanitation Collaborative Council; 2016.

2. Kar K, Chambers R. Handbook on Community-led Total Sanitation. London, UK: Plan International; 2008.

3. Ministry of Water and Environment [MWE]. Uganda Water and Environment Sector Performance Report 2015. Kampala, Uganda.

4. Ministry of Water and Environment [MWE]. Uganda Water and Environment Sector Performance Report 2019. Kampala, Uganda.

5. Water and Sanitation Program [WSP], 2012. Africa: Economic of sanitation initiative. http://www.wsp.org/wsp/content/africa-economic-impacts-sanitation Accessed 15 Feb $\underline{2021}$. 
6. Greaves F. 'CLTS in Post-Emergency and Fragile States Settings', Frontiers of CLTS: Innovations and Insights 9, Brighton: IDS, 2016.

7. USAID. An Examination of CLTS's Contributions toward Universal Sanitation. USAID, Washington, DC, 2018.

8. World Health Organization [WHO]. Guidelines on sanitation and health. 2018.

9. Robinson A. Final Evaluation: Pan African CLTS program 2010-2015; Plan Netherlands, 2016.

10. USAID/LEARN. Deep Dive Of Akros Community-Led Sanitation Program In Zambia, 2018.

11. Garn J V, Sclar G D, Freeman M C, Penakalapati G, Alexander K T, Brooks P. The impact of sanitation interventions on latrine coverage and latrine use: A systematic review and meta-analysis. Int. J. Hyg. Environ. Health 2017; 220, 329.10.1016/j.ijheh.2016.10.001.

12. Harter M, Inauen J, Mosler H J. How does Community-Led Total Sanitation (CLTS) promote latrine construction, and can it be improved? A cluster-randomized controlled trial in Ghana, Social Science \& Medicine, Volume 245, 2020, 112705, ISSN 0277-9536, https://doi.org/10.1016/j.socscimed.2019.112705. 